\title{
Metabolomics of Selenium: Se Metabolites Based on Speciation Studies
}

\author{
Kazuo T. Suzuki* \\ Department of Toxicology and Environmental Health, Graduate School of Pharmaceutical Sciences, Chiba University, 1-8-1 Inohana, \\ Chuo, Chiba 260-8675, Japan
}

(Received December 9, 2004)

\begin{abstract}
Selenium is a trace element essential for the normal function of the body. This metalloid is quite unique in its metabolism compared with typical essential metals such as copper and zinc. In the present communication, the metabolism of selenium in the body was reviewed from the viewpoint of metabolomics based on speciation studies. Both inorganic and organic forms of slenium can be the nutritional source, and they are transformed to the common intermediate, selenide or its equivalent. Selenite and selenate are reduced simply to selenide for further utilization and/or excretion. On the other hand, organic selenocysteine is directly lysed to selenide, while selenomethionine is transformed to selenocysteine (trans-selenation pathway), similarly to the trans-sulfuration pathway for methionine to cysteine, and then lysed to selenide. Selenide is known to be transformed to selenocysteine on tRNA, and the selenocysteinyl residue is incorporated into selenoprotein sequences by the codon specific to selenocysteine, UGA. Diverse selenium chemicals in foods seem to be recognized as selenium species and transformed to selenide, and then utilized for the synthesis of selenoproteins. Surplus selenium is methylated stepwise to methylated selenium metabolites from the common intermediate selenide. The major urinary metabolite is $1 \beta$-methylseleno- $N$-acetyl-Dgalactosamine (selenosugar). Trimethylselenonium has been recognized as the urinary metabolite excreted in response to excessive doses and as a biological marker for excessive doses. However, recent results contradicted this.
\end{abstract}

Key words — selenium, metabolomics, speciation, metallome, selenite, selenosugar

\section{INTRODUCTION}

Selenium is a metalloid belonging to the same group on the periodic table as oxygen, sulfur and tellurium, i.e., group 16, and is a trace bio-element essential for the normal function of the body, being present at around $10 \mathrm{mg} \mathrm{Se} / 60 \mathrm{~kg}$ body weight. Selenium is similar to sulfur in chemical property, and has to be discriminated biologically from abundant sulfur during its metabolism in the body. At the same time, selenium is known to be a highly toxic element, with a narrow adequate range between deficient and excessive doses, being one order of the range $(0.1-1.0 \mu \mathrm{g} / \mathrm{g}$ diet or $\mathrm{ml}$ drinking water) in experimental animals.

\footnotetext{
*To whom correspondence should be addressed: Department of Toxicology and Environmental Health, Graduate School of Pharmaceutical Sciences, Chiba University, 1-8-1 Inohana, Chuo, Chiba 260-8675, Japan. Tel. \& Fax: +81-43-226-2865; E-mail: ktsuzuki@p.chiba-u.ac.jp
}

Selenium is essential for the body with that it forms the active center for seleno-enzymes that carry out redox reactions such as glutathione peroxidase (GPx), thioredoxin reductase, thyroid hormone deiodinase families and so on. ${ }^{1-6)}$ However, while selenium does form an active center in the formation of a selenol group (-SeH) on selenocysteinyl (SeCys) residues in selenoprotein sequences (direct gene product), it does not do so in the form coordinated with functional groups on polypeptide sequences, as in the case for typical metalloproteins (enzyme products). As typically demonstrated above, selenium shows quite a different metabolism from that of typical metals. The present communication introduces current knowledge of the metabolism of selenium based on speciation studies. 


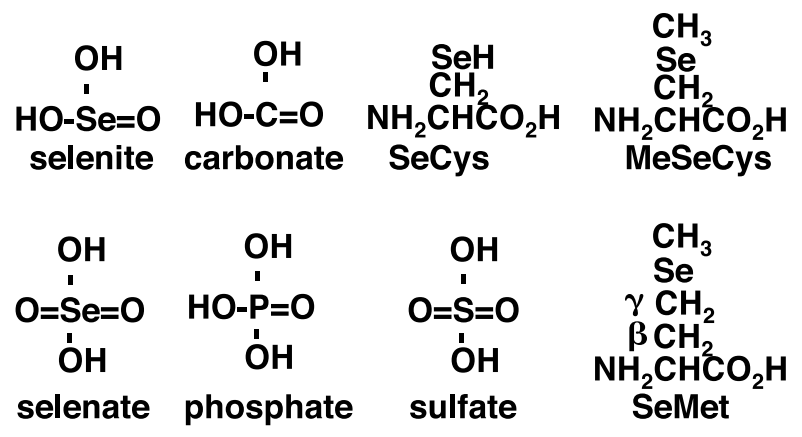

Fig. 1. Structures of Inorganic Selenium Compounds, the Related Oxyanions, and Organic Selenium Compounds

Selenocysteine, SeCys; Se-methylselenocysteine, MeSeCys; selenomethionine, SeMet.

\section{NUTRITIONAL SOURCE OF SELENIUM}

Selenium is a metalloid and can be present in inorganic forms, a metallic form $\left(\mathrm{Se}^{0}\right)$ and oxyanions such as selenite $\left(\mathrm{SeO}(\mathrm{OH})_{2}\right)$ and selenate $\left(\mathrm{SeO}_{2}(\mathrm{OH})_{2}\right)$, and also in organic forms, as selenoamino acids such as SeCys and selenomethionine (SeMet) (Fig. 1). Selenite and selenate are well known inorganic selenium sources, while SeCys and SeMet residues in proteins are most common organic selenium sources in foods (Fig. 2). ${ }^{1)}$ Namely, SeCys is present as an amino acid residue in selenoproteins (seleno-enzymes) in plants and animals (vegetables and meats in foods), which is incorporated into amino acid sequences of selenoproteins by the specific codon to SeCys residue, as described in the next section. On the other hand, SeMet is present in the form of SeMet residue in general proteins without being discriminated from methionine (Met), as described later (p. 111).

SeMet is also present in free SeMet, especially in a selenium accumulator. ${ }^{7)}$ A methylated form of SeCys, Se-methyl selenocysteine (MeSeCys) is also present in selenim accumulators in the forms of free and $\gamma$-glutamyl-Se-methylselenocysteine. This is because free selenol (-SeH) groups are more highly reactive than free mercapto (-SH) groups, and free SeCys is too reactive to be present in the free form. Instead, selenium accumulates in accumulators in the form of non-reactive amino acids and peptides $\left(-\mathrm{SeCH}_{3}\right.$ group rather than $-\mathrm{SeH}$ group) such as MeSeCys and SeMet, and $\gamma$ glutamyl-Se-methylselenocysteine. ${ }^{7)}$

Selenium-accumulating plants can be divided into three groups: selenite-accumulators (broccoli

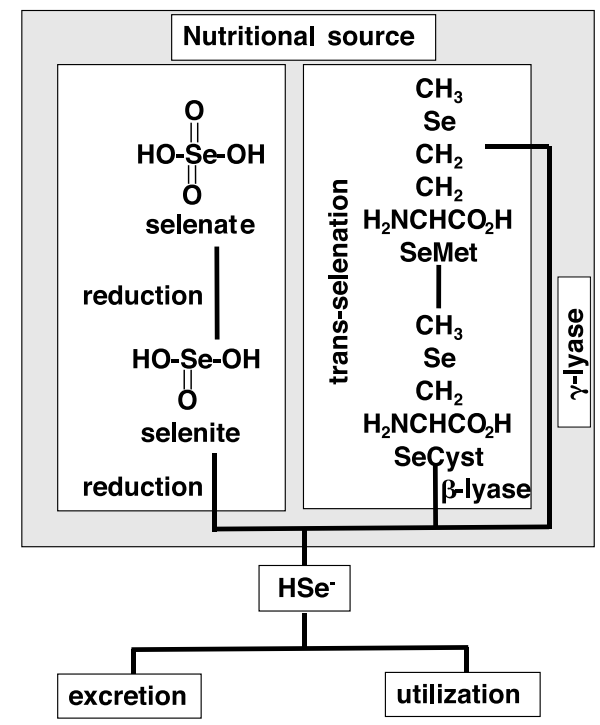

Fig. 2. Inorganic and Organic Forms of Selenium Compounds are Transformed to the Assumed Common Intermediate Selenide for the Utilization and Excretion of Selenium Selenite and selenate are reduced simply to selenide. On the other hand, selenocysteine is lysed at the $\beta$-position to give selenide, and selenomethionine is transformed to selenocysteine through the transselenation pathway or directly lysed at the $\gamma$-position to give selenide. Selenide is utilized for the synthesis of selenoproteins or excreted after being methylated stepwise. Thus, selenide is the common intermediate for nutritional selenium and at the same time the checkpoint metabolite for utilization and excretion.

and cucumber), SeMet-accumulators (grains such as wheat, and mushroom) and MeSeCys-accumulators (garlic and onion). ${ }^{7)}$

The most common selenium source in normal foods is thus selenium bound to proteins: SeCys in seleno-enzymes, and SeMet in general proteins in foods (vegetables and meats) (Fig. 2), followed by selenite and selenate in drinking water and foods. On the other hand, free selenite, SeMet and MeSeCys are abundant in accumulator plants and yeasts, as mentioned above.

Many minor metabolites other than SeCys and SeMet are present in foods originating from plants and yeasts, especially those grown in selenium-fortified (selenium-enriched) soils and media, i.e., selenized plants, mushrooms and yeasts, and their identification is one of the targets for speciation studies.

\section{SELENIUM IN PROTEINS}

Selenium is present in proteins in the forms of either SeCys or SeMet residues (Fig. 3). Although 


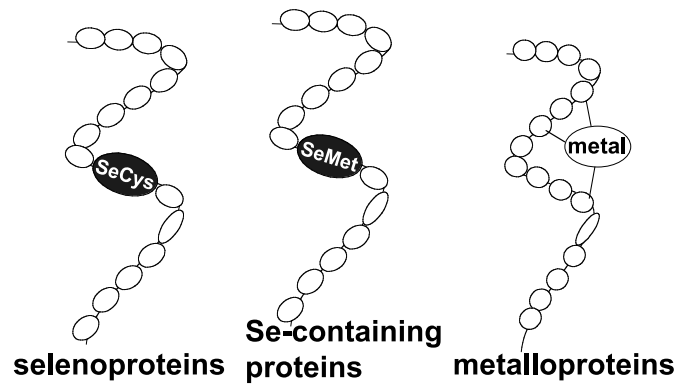

Fig. 3. Difference in the Structure of Metal/Metalloid-Protein Complexes between Selenium and Typical Metals

Selenium is present in proteins in either the selenocysteinyl or selenomethionyl residue form (direct gene products), while typical metals such as copper and zinc are coordinated to functional groups on proteins (enzyme products)

SeMet of exogenous sources can be incorporated in its intact form into proteins by the Met codon without being distinguishing between SeMet and Met, i.e., AUG codon, ${ }^{8,9)} \mathrm{SeCys}$ residues incorporated into selenoproteins are not of an exogenous intact one but of endogenously prepared SeCys residue, which is synthesized from the activated selenium, selenophosphate ${ }^{10)}$ and activated serine (Ser) resi-

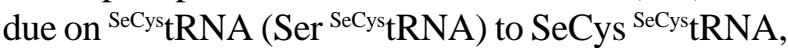
as demographically drawn in Fig. 4. ${ }^{1,11,12)}$

The codon for the incorporation of SeCys residues is UGA that is the stop codon in general. Five components are known to be required for the translation of UGA codon to SeCys residue in selenoproteins: two cis-sequences, a SeCys-insertion sequence (SECIS) element in the 3'-untranslated region and a SeCys codon (UGA) in the coding region, and three known trans-acting factors, a SeCysspecific translation elongation factor (EFSeCys), the SeCys ${ }^{S e C y s}$ tRNA, and a SECIS-binding protein (SBP2), ${ }^{13)}$ as schematically drawn in Fig. 4. Therefore, selenium in the form of SeCys residue can be incorporated into proteins after being recognized as selenium species at the selenide level, while selenium in the form of SeMet is incorporated without being recognized as selenium species, i.e., AUG codon (Fig. 5). Proteins containing selenium in the form of SeCys are called selenoproteins, while proteins containing selenium in the form of SeMet are called selenium-containing proteins but not selenoproteins (Fig. 3). Selenium present in SeMet can be recognized as selenium species during the trans-selenation pathway, similarly to the transsulfuration pathway for Met to Cys through cystathionine (Fig. 5), as described in the following section.
Thus, selenium in proteins is present as a direct gene product in the forms of SeCys and/or SeMet residues, and it is completely different from other metals/metalloids, where metals/metalloids are bound to proteins in the form coordinated to functional groups on proteins (metal-protein complexes), as schematically drawn in Fig. 3.

\section{METABOLIC PATHWAY LEADING TO THE COMMON INTERMEDIATE, SELENIDE}

Diverse inorganic and organic selenium compounds are utilized as the nutritional source of selenium, suggesting that these selenium chemicals are transformed to the common intermediate, and then utilized for the synthesis of SeCys ${ }^{\mathrm{SeC} y}$ tRNA to meet to the SeCys codon for selenoprotein synthesis (Fig. 2) ${ }^{11-13)}$ Although the assumed common intermediate, selenide, or its equivalent has been proposed for all selenium chemicals that can be utilized for the synthesis of selenoproteins as described above and in Fig. 1, selenide is a highly reactive compound and it may not be present in a free form. In fact, selenide is readily bound to proteins when it is incubated in the liver supernatant (unpublished observation). Therefore, the assumed intermediate is called selenide or its equivalent in this communication.

\section{Transformation of Inorganic Selenium Com- pounds to Selenide}

Inorganic selenium species, selenite and selenate, can be reduced simply to selenide. Although selenate is not readily reduced to selenite by glutathione (GSH), and more rigorous reducing conditions are required, selenite is readily reduced by GSH to the assumed intermediate selenide.

Ionic selenium ions, selenite and selenate, follow bicarbonate and phosphate, respectively, in their transport in the body because of their similar ionic forms (Fig. 1). In fact, selenite ions in the bloodstream are readily taken up by red blood cells (RBCs) through band 3 protein without being excreted into urine, ${ }^{14)}$ while selenate ions are not taken up by RBCs but directly taken up by hepatocytes through the transport system for phosphate, and partly excreted directly into urine. ${ }^{15)}$ Selenite taken up by RBCs is readily reduced to selenide, and then effluxed into the bloodstream in the presence of albumin and transferred to the liver in the form bound to albumin, ${ }^{16}$ as demographically drawn in Fig. 6. Thus, selenide 


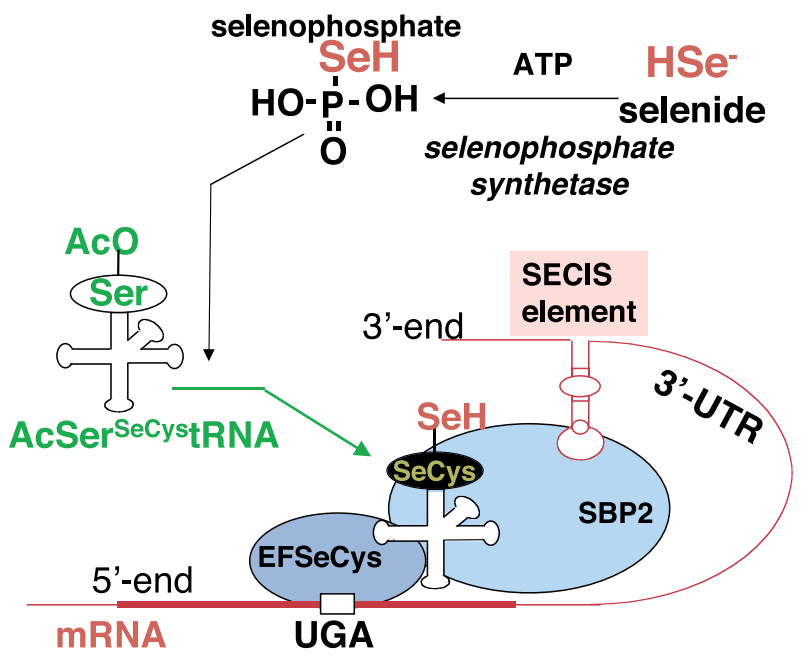

Fig. 4. Demographic Translation Mechanism for the Synthesis of Selenoproteins

Two cis-sequences, a SECIS element in the $3^{\prime}$-untranslated region and a SeCys codon (UGA) in the coding region, and three trans-acting factors, a SeCys-specific translation elongation factor (EFSeCys), the

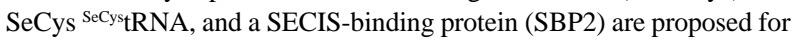
the translation of the UGA codon to the SeCys sequence.

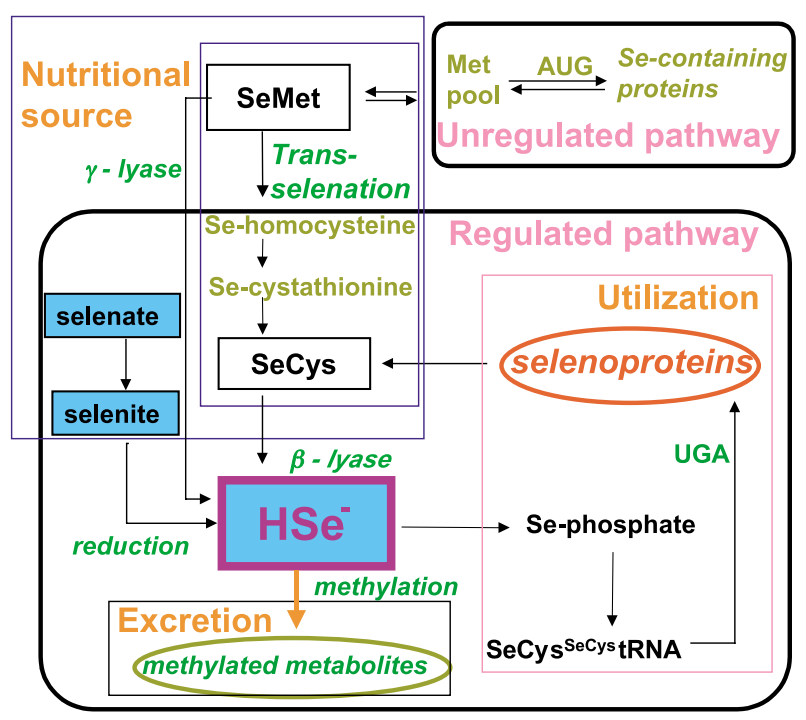

Fig. 5. Metabolic Pathway for Selenium

Once selenium compounds are recognized as selenium species, they are transformed to the common intermediate metabolite selenide, and then utilized for the synthesis of selenoproteins or excreted after being methylated stepwise. Selenomethionine is an exceptional selenium compound, i.e., when recognized as a selenium species, it can be transformed to selenocysteine through the trans-selenation pathway and then lysed by $\beta$-lyase or directly by $\gamma$-lyase to selenide. However, at the same time, selenomethionine can be utilized for the synthesis of proteins without the body distinguishing between selenomethionine and methionine (unregulated pathway).

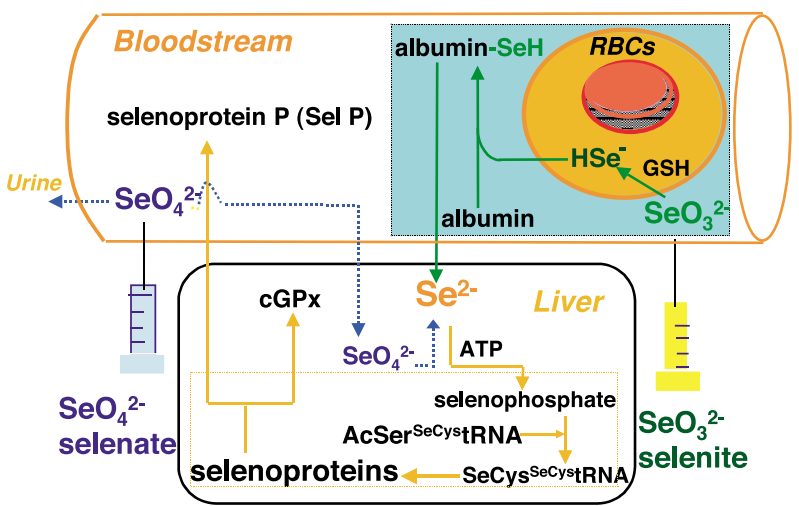

Fig. 6. Difference in the Metabolic Pathway between Selenite and Selenate in the Bloodstream

Selenite is efficiently taken up by red blood cells and reduced to selenide, effluxed into the plasma with the aid of albumin, and then transferred in the form bound to albumin to the liver. On the other hand, selenate is transferred directly to the liver, and partly excreted directly into urine.

of selenite origin and selenate are taken up differently by the liver and utilized for the synthesis of selenoproteins P (Sel P) and cellular GPx (cGPx), the former selenoproteins being synthesized more efficiently than the latter one and excreted into the bloodstream. ${ }^{17)}$

\section{Transformation of Organic Selenium Com- pounds to Selenide}

Contrary to rather simple reduction reactions for inorganic selenium compounds, organic selenium compounds have to be transformed to selenide through a more sophisticated pathway. Organic selenium chemicals can be oxidized to selenate or selenite. However, nutritional sources of organic selenium compounds, mostly selenoamino acids, are believed to be transformed to selenide through reductive cleavage of the $\mathrm{C}$-Se bond by lyase reactions. ${ }^{18-22)}$ In the case of SeCys, $\beta$-lyase directly produces selenide, and this $\beta$-lyase reaction is thought to be the main route for the transformation of selenoamino acids to selenide. In fact, SeMet is also believed to be transformed to SeCys through the trans-selenation pathway (SeMet $\rightarrow$ Se-adenosyl SeMet $\rightarrow$ Se-adenosylselenohomocysteine $\rightarrow$ selenohomocysteine $\rightarrow$ selenocystathionine $\rightarrow$ SeCys), similarly to the trans-sulfuration pathway (Met $\rightarrow$ adenosyl SeMet $\rightarrow$ adenosylhomocysteine $\rightarrow$ homocysteine $\rightarrow$ cystathionine $\rightarrow$ Cys).

The direct C-Se cleavage at the $\gamma$ position of SeMet, i.e., $\gamma$-lyase reaction, is proposed to be working in the case of excessive selenium intake. ${ }^{23)}$ It is 
also proposed that $\gamma$-lyase functions to produce the assumed reactive selenium species, methylselenol $\left(\mathrm{CH}_{3} \mathrm{SeH}\right)$ for SeMet, while $\beta$-lyase functions to produce methylselenol from MeSeCys. SeMet and $\mathrm{MeSeCys}$ are assumed to produce methylselenol in the metabolism of their anti-cancinogenic agents. ${ }^{24-28)}$

\section{REGULATED AND NON-REGULATED PATHWAYS FOR SELENOMETHIONINE}

Although SeCys is incorporated into selenoproteins through the specific codon to SeCys (UGA), SeMet does not have a specific codon and is incorporated into general proteins by the same AUG codon as that to Met (Fig. 5). ${ }^{8,9)}$ Thus, SeMet is not recognized as a selenium species during the incorporation into proteins, suggesting that general proteins contain SeMet depending on the SeMet/Met ratio in foods. ${ }^{29)}$ It also means that SeMet in a nutritional source can be stored in the body in the form incorporated in general proteins. Therefore, selenium in the form of SeMet in general proteins seems to be a stable and safe storage mode for selenium. Although selenium in this storage is not recognized as selenium species, once it is liberated to free SeMet during the degradation of proteins, it can be recognized as selenium species through the transselenation pathway or through the direct lyase reaction at the $\gamma$-position (Figs. 2 and 5).

When excessive SeMet is given to experimental animals, it is incorporated into proteins at a higher ratio than the normal one depending on the elevated SeMet/Met ratio in foods, i.e., (excessive SeMet + SeMet in foods)/(Met in foods). SeMet incorporated into non-regenerating proteins, such as those in hair and red blood cells, is retained at the ratio of SeMet/ Met when those proteins were synthesized, while SeMet incorporated into actively regenerating proteins can be removed during the degradation and resynthesis of proteins, and then transformed to selenide for utilization or excretion. This speculation, i.e., the regulated and non-regulated pathway for SeMet (Fig. 5), was demonstrated in rats by feeding SeMet, i.e., selenium concentrations in hair and RBCs were more elevated by feeding SeMet than the concentrations in livers and kidneys. ${ }^{29}$

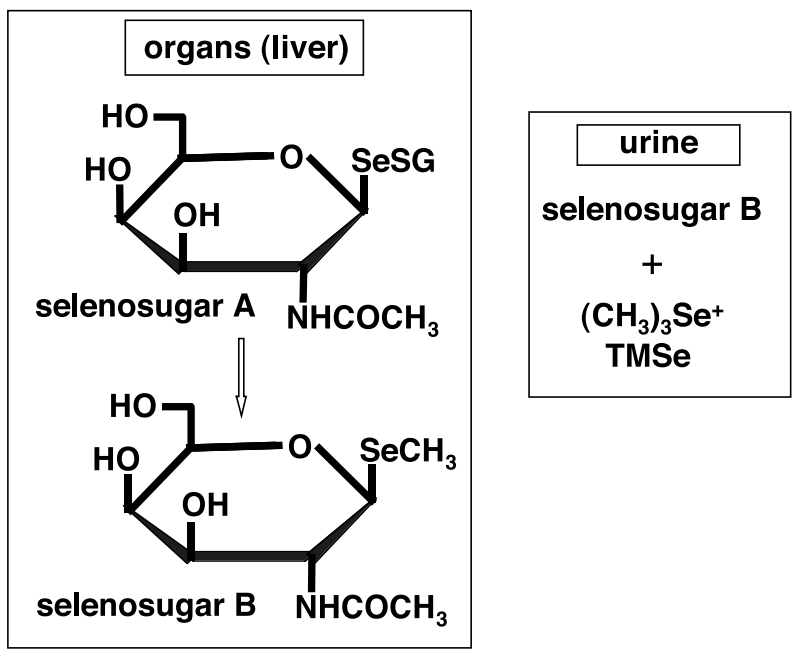

Fig. 7. Transformation of Selenosugar in Organs (Liver) and Excretion into Urine, together with Trimethylselenonium

An activated selenium metabolite such as GS-SeH seems to be transferred to the sugar moiety of selenosugar to form selenosugar-A, and then it is methylated to form selenosugar-B in the liver and other organs. Selenosugar-B is the form excreted into urine, together with trimethylselenonium.

\section{EXCRETION OF SELENIUM}

Once selenium is taken up by the body, it is mostly excreted into urine. Therefore, the amount of selenium in urine depends on the dose in the nutritional range. However, excessive selenium is excreted not only into urine but also into breath (exhaled into breath). ${ }^{30-32)}$ In either case, selenium is excreted after being methylated stepwise. Urinary metabolites are known to be monomethylated selenium and trimethylselenonium (TMSe), while selenium is exhaled in the form of dimethylselenide. ${ }^{31}$ The ratio of the two major selenium metabolites in urine changes depending on the selenium dose, i.e., at a lower dose, selenium is excreted mostly in one form (monomethylated selenium), while at a higher dose, in the two forms (the trimethylated form (TMSe) increases with doses). ${ }^{33-35)}$

The monomethylated selenium in urine was originally thought to be methylselenol $\left(\mathrm{CH}_{3} \mathrm{SeH}\right) .{ }^{35}$, However, it was finally identified to Se-methyl- $N$ acetylgalactosamine (selenosugar), as shown in Fig. 7. ${ }^{36)}$ Two selenosugars have been identified in the liver, selenosugar-A and -B. Selenosugar-A was thought to be the precursor of selenosugar $B$, and identified as Se-glutathionyl- $N$-acetylgalactosamine, while selenosugar-B was identical as that of the urinary selenosugar. ${ }^{36,37)}$ As selenium is an essential el- 
ement and selenoproteins are presumed to be synthesized and then degraded in each cell, selenosugars seem to be produced in each cell as observed in the liver, and selenosugar-B is presumed to be excreted from each cell into the bloodstream, and then into urine.

\section{TOXICOLOGICAL AND NUTRITIONAL MARKERS}

Selenium is an essential element. Therefore, its concentration is homeostatically regulated within the adequate range between deficiency and excess. Selenium is excreted mostly into urine for a dose lower than the excessive one, and the major selenium metabolite is selenosugar. ${ }^{36,38)}$ The amount of selenosugar in urine depends linearly on the selenium dose, and then reaches a plateau at a selenium concentration higher than $2.0 \mu \mathrm{g} / \mathrm{g}$ diet or ml drinking water, which is supposed to be the toxic dose. With selenosugar reaching the plateau, TMSe starts to increase in rats. ${ }^{35,37)}$ Therefore, selenosugar was assumed to be a nutritional marker increasing with doses up to the lower toxic dose, while TMSe may be a nutritional marker for the excessive dose, and also a toxicological marker. However, recent results with adult and young rats suggested that the relation between selenium dose and TMSe/selenosugar ratio could be applicable only to young rats. ${ }^{39)}$ Namely, although adult rats are more sensitive to excessive selenium dose, their urinary metabolite was mostly selenosugar. These observations indicate that urinary metabolites, selenosugar and TMSe may not be used as nutritional and toxicological markers except that selenium is excreted in the form of selenosugar depending on the dose within the low toxic dose. ${ }^{39)}$

\section{SPECIATION OF METALLOME IN THE METABOLOMICS OF SELENIUM}

The expression of biological roles of selenium is certainly related to its metabolism as an essential nutrient, and in some cases as an excessive toxic element, and hence to the metabolites and chemical reactions in relation to the metabolism. As diverse selenium compounds can be a nutritional source and their chemical structures affect their absorption and distribution, selenium in foods is currently a target for speciation studies, as also referred to in this communication. ${ }^{3,726)}$ Selenium in a biological system is present in diverse chemical forms, even if a single chemical selenium species is administered into it.

The entirety of selenium species in a biological system can be defined as a metallome, ${ }^{40,41)}$ and metallome in a biological system can be speciated by fractionating (by separating and detecting) all species of a metal. ${ }^{42)}$ Thus, speciation studies of a metallome clarify all species of a metal in a biological system at a specific time point, thereby giving a static aspect of the metabolic pathway of a metal. The dynamic aspect of the metabolic pathway, i.e., the metabolism of a metal, can be obtained by speciating the metallome that is changing with time. Therefore, speciation studies are essential to understanding a metallome in a biological system, and the dynamic aspect of the metabolism, metabolomics. ${ }^{40-43)}$

Speciation study of selenium in foods can give a static aspect of all selenium species in foods. However, once selenium is administered as a food material into a biological system, such as the whole body of a rat, it starts to interact with various biological constituents depending on its selenium species and to be distributed among organs, within cellular organelle, and then to be transformed to various metabolites leading to metabolism. Therefore, metabolomics based on speciation study is essential to understanding a metal in a biological system, especially for selenium because selenium itself is transformed to inorganic and organic species for metabolism.

\section{METABOLOMICS TO METALLOMICS}

Selenium species in foods and selenium metabolites in a biological system can be speciated currently by a hyphenated technique, HPLC-ICP MS, as also introduced in the present communication. Speciation studies were effective for selenium and gave information as to its chemical species in foods and about metabolites such as urinary metabolites that are not attainable by other approaches. However, speciation studies have not been applied efficiently to metals such as copper and zinc bound to proteins, in which those metals are bound always to proteins.

Selenium expresses its biological roles in the form of selenoproteins (seleno-enzymes) which participate in the normal function of the body. However, excessive selenium seems to express its toxicity by unregulated redox reactions occurring from selenium metabolites rather than as the result of ex- 
cessive activities of selenoproteins (seleno-enzymes).

Further sophisticated speciation techniques will be required to clarify the metabolism and biological significance of selenium leading to metallomics. ${ }^{40-43)}$

Acknowledgements The author wishes to acknowledge the Grant-in-Aid from the Ministry of Education, Culture, Science, Sports and Technology, Japan (No. 16209004).

\section{REFERENCES}

1) Suzuki, K. T. and Ogra, Y. (2002) Metabolic pathway for selenium in the body: Speciation by HPLC-ICP MS with use of enriched Se. Food Addit.Contam., 19, 974-983.

2) Kohrle, J. (2004) Selenium in biology and medicine - further progress and increasing interest. J. Trace Elem. Med. Biol., 18, 61-63.

3) Whanger, P. D. (2002) Selenocompounds in plants and animals and their biological significance. J. Am. Coll. Nutr., 21, 223-232.

4) Bock, A. (2000) Biosynthesis of selenoproteins an overview. Biofactors, 11, 77-78.

5) Bock, A., Forchhammer, K., Heider, J. and Baron, C. (1991) Selenoprotein synthesis: an expansion of the genetic code. Trends Biochem. Sci., 16, 463-467.

6) Burk, R. F. and Hill, K. E. (1993) Regulation of selenoproteins. Rev. Nutr. Аппи., 13, 65-81.

7) Ogra, Y., Ishiwata, K., Encinar, J. R., Lobinski, R. and Suzuki, K. T. (2004) Speciation of selenium in selenium-enriched shiitake mushroom, Lentinula edodes. Anal. Bioanal. Chem., 379, 861-866.

8) McConnell, K. P. and Hoffman, J. L. (1972) Methionine-selenomethionine parallels in rat liver polypeptide chain synthesis. FEBS Lett., 24, 60-62.

9) Butler, J. A. and Whanger, P. D. (1989) Influence of dietary methionine on the metabolism of selenomethionine in rat. J. Nutr., 119, 1001-1009.

10) Chambers, I., Framoton, J., Goldfarb, P., Affara, N., McBain, W. and Harrison, P. R. (1986) The structure of the mouse glutathione peroxidase gene: the selenocysteine in the active site is encoded by the 'termination' codon, TGA. EMBO J., 5, 1221-1227.

11) Veres, Z., Tsai, L., Scholz, T. D., Politino, M., Balaban, R. S. and Stadtman, T. C. (1992) Synthesis of 5-methylaminomethyl-2-selenouridine in tRNAs: ${ }^{31} \mathrm{P}$ NMR studies show the labile selenium donor synthesized by the selD gene product contains selenium bonded to phosphorus. Proc. Natl. Acad.
Sci. U.S.A., 89, 2975-2979.

12) Glass, R. S., Singh, W. P., Yung, W., Veres, Z., Scholz, T. D. and Stadtman, T. C. (1993) Monoselenophosphate: Synthesis, characterization, and identity with the prokaryotic biological selenium donor, compound SePX. Biochemistry, 32, 1255512559.

13) Mehta, A., Rebsch, C. M., Kinzy, S. A., Fletcher, J. E. and Copeland, P. R. (2004) Efficiency of mammalian selenocysteine incorporation. J. Biol. Chem., 279, 37852-37859.

14) Suzuki, K. T., Shiobara, Y., Itoh, M. and Ohmichi, M. (1998) Selective uptake of selenite by red blood cells. Analyst, 123, 63-67.

15) Kobayashi, Y., Ogra, Y. and Suzuki, K. T. (2001) Speciation and metabolism of selenium in rats injected with ${ }^{82} \mathrm{Se}$-enriched selenate and selenite in rats. J. Chromatogr. B, 760, 73-81.

16) Shiobara, Y. and Suzuki, K. T. (1998) Binding of selenium (administered as selenite) to albumin after efflux from red blood cells. J. Chromatogr. B, 710, 49-56.

17) Suzuki, K. T., Ishiwata, K. and Ogra, Y. (1999) Incorporation of selenium into selenoprotein $\mathrm{P}$ and extracellular glutathione peroxidase: HPLC-ICP MS data with enriched selenite. Analyst, 124, 17491753.

18) Esaki, N., Nakamura, T., Tanaka, H., Suzuki, T., Morino, Y. and Soda, K. (1981) Enzymatic synthesis of selenocysteine in rat liver. Biochemistry, 20, 4492-4496.

19) Esaki, N., Nakamura, T., Tanaka, H., Suzuki, T. and Soda, K. (1982) Selenocysteine lyase, a novel enzyme that specifically acts on selenocysteine. $J$. Biol. Chem., 257, 4386-4391.

20) Ganther, H. E. (1986) Pathways of Se metabolism including respiratory excretory products. J. Am. Coll. Toxicol., 5, 1-5.

21) Schomburg, L., Schweizer, U. and Kohrle, J. (2004) Selenium and selenoproteins in mammals: extraordinary, essential, enigmatic. Cell. Mol. Life Sci., 61, 1988-1995.

22) Schrauzer, G. N. (2003) The nutritional significance, metabolism and toxicology of selenomethionine. Adv. Food Nutr. Res., 47, 73-112.

23) Okuno, T., Kubota, T., Kuroda, T., Ueno, H. and Nakamuro, K. (2001) Contribution of enzymic $\alpha, \gamma$ elimination reaction in detoxification pathway of selenomethionine in mouse liver. Toxicol. Appl. Pharmacol., 176, 18-23.

24) Dong, Y., Zhang, H., Hawthorn, L., Ganther, H. E. and Ip, C. (2003) Delineation of the molecular basis for selenium-induced growth arrest in human prostate cancer cells by oligonucleotide array. 
Cancer Res., 63, 52-59.

25) Ip, C., Dong, Y. and Ganther, H. E. (2002) New concepts in selenium chemoprevention. Cancer Metastatis. Rev., 21, 281-289.

26) Ip, C., Birringer, M., Block, E., Kotrebai, M., Tyson, J. F., Uden, P. C. and Lisk, D. J. (2000) Chemical speciation influences comparative activity of selenium-enriched garlic and yeast in mammary cancer prevention. J. Agric. Food Chem., 48, 20622070.

27) Dong, Y., Lisk, D., Block, E. and Ip, C. (2001) Characterization of the biological activity of $\gamma$ glutamyl-Se-methylselenocysteine: A novel, naturally occurring anticancer agent from garlic. Cancer Res., 61, 2923-2928.

28) Seo, Y. R., Kelley, M. R. and Smith, M. (2002) Selenomethionine regulation of p53 by a ref1dependent redox mechanism. Proc. Natl. Acad. Sci. U.S.A., 99, 14548-14553.

29) Shiobara, Y., Yoshida, T. and Suzuki, K. T. (1998) Effects of dietary selenium species on Se concentrations in hair, blood and urine. Toxicol. Appl. Pharmacol., 152, 309-314.

30) McConnell, K. P. and Roth, D. M. (1966) Respiratory excretion of selenium. Proc. Soc. Exp. Biol. Med., 123, 919-921.

31) McConnell, K. P. and Portman, O. W. (1952) Toxicity of dimethylselenide in the rat and mouse. Proc. Soc. Exp. Biol. Med., 79, 230-231.

32) Hsieh, H. S. and Ganther, H. E. (1977) Biosynthesis of dimethylselenide from sodium selenite in rat liver and kidney cell-free systems. Biochem. Biophys. Acta, 497, 205-217.

33) Byard, J. L. (1969) Trimethylselenide. A urinary metabolite of selenite. Arch. Biochem. Biophys., 130, 556-560.

34) Palmer, I. S., Fischer, D. D., Halverson, A. W. and
Olson, O. E. (1969) Identification of a major selenium excretory product in rat urine. Biochim. Biophys. Acta, 177, 336-342.

35) Itoh, M. and Suzuki, K. T. (1997) Effects of dose on the methylation of selenium to monomethylselenol and trimethylselenonium ion in rats. Arch. Toxicol., 71, 461-466.

36) Kobayashi, Y., Ogra, Y., Ishiwata, K., Takayama, H., Aimi, N. and Suzuki, K. T. (2002) Selenosugars are key and urinary metabolites for selenium excretion within the required to low-toxic range. Proc. Natl. Acad. U.S.A., 99, 15932-15936.

37) Ogra, Y., Ishiwata, K., Takayama, H., Aimi, N. and Suzuki, K. T. (2002) Identification of a selenium metabolite, Se-methyl-N-acetyl-selenohexosamine, in rat urine by the HPLC-ICP MS and -ESI MS/MS methods. J. Chromatogr. B, 767, 301-312.

38) Francesconi, K. A. and Pannier, F. (2004) Selenium metabolites in urine: a critical overview of past work and current status. Clin. Chem., 50, 2240-2253.

39) Suzuki, K. T., Kurasaki, K., Okazaki, N. and Ogra, Y. (2005) Selenosugar and trimethylselenonium among urinary Se metabolites: dose- and age-related changes. Toxicol. Appl. Pharmacol., in press.

40) Szpunar, J. (2004) Metallomics: a new frontier in analytical chemistry. Anal. Bioanal. Chem., 378, 5456.

41) Suzuki, K. T. (2004) Metabolomics of arsenic based on speciation studies. Anal. Chem. Acta, in press.

42) Templeton, D. M., Ariese, F., Cornelis, R., Danielsson, L., Muntau, H., Van Leeuwen, H. P. and Lobinski, R. (2000) Guidelines for terms related to chemical speciation and fractionation of elements. Definitions, structural aspects, and methodological approaches. Pure Appl. Chem., 72, 1453-1470.

43) Haraguchi, H. (2004) Metallomics as integrated biometal science. J. Anal. At. Spectrom., 19, 5-14. 